Check for updates

Cite this: RSC Adv., 2020, 10, 38617

Received 17th June 2020

Accepted 15th September 2020

DOI: $10.1039 / \mathrm{dOra07482a}$

rsc.li/rsc-advances
Iron oxide and various metal oxide nanotubes engineered by one-pot double galvanic replacement based on reduction potential hierarchy of metal templates and ion precursors $\dagger$

\author{
Aloka Paragodaarachchi,,$^{\text {ab }}$ Steven Medvedovsky, $\dot{t}^{\mathrm{a}}$ Justin Fang, ${ }^{\text {ab }}$ Timothy Lau ${ }^{\mathrm{a}}$ \\ and Hiroshi Matsui ${ }^{\star a b c d}$
}

A one-pot double galvanic approach was explored for the rational synthesis of metal oxide nanotubes, predictable based on the reduction potential hierarchy of templates and ion precursors (e.g., Ag nanowire substrate is oxidized by $\mathrm{MnO}_{4}{ }^{-}$ions and it is consecutively reduced by $\mathrm{Fe}^{2+}$ ions to form an $\mathrm{Fe}_{2} \mathrm{O}_{3}$ nanotube). This method generated a variety of metal oxide nanotubes via a redox potential landscape.

\begin{abstract}
A variety of inorganic nanoparticles with different shapes and sizes have been synthesized since these parameters could be used for fine-tuning their physical and biological properties. ${ }^{1}$ Among them, one dimensional (1D) nanostructures are pivotal due to their characteristic electrical, magnetic and chemical properties. ${ }^{2,3}$ Free standing hollow 1D metal oxide nanoparticles represent an intriguing class of nanomaterials due to their high surface to volume ratio and low density, ${ }^{4,5}$ leading to distinct properties in catalysis, ${ }^{6}$ energy storage, ${ }^{7}$ gas sensors, ${ }^{8}$ photodetectors, ${ }^{2}$ and in biomedical applications such as drug delivery. ${ }^{9}$

Previously, metal oxide nanotubes were synthesized by techniques such as template-mediated and template-free methods which include hydrothermal reaction, and chemical etching method., ${ }^{4,10}$ In the template-mediated method, posttreatment is necessary to remove templates. Thus, it adds complexity to the synthesis process, increases frequency of structural deformation, and introduces more impurities even after undergoing a separation process for the removal of leftover template in the sample. ${ }^{7}$ Moreover, the size of the nanotube is also limited by the size of the template, which is often larger than $200 \mathrm{~nm} .{ }^{5}$ Hydrothermal synthesis of metal oxide nanotubes has disadvantages such as difficulty of controlling
\end{abstract}

\footnotetext{
${ }^{a}$ Department of Chemistry, Hunter College, City University of New York, 695 Park Avenue, New York, NY 10065, USA. E-mail: hmatsui@hunter.cuny.edu

${ }^{b}$ PhD Program in Chemistry, The Graduate Center of the City University of New York, New York, NY 10016, USA

'PhD Program in Biochemistry, The Graduate Center of the City University of New York, New York, NY 10016, USA

${ }^{d}$ Department of Biochemistry, Weill Cornell Medicine, 413 East $69^{\text {th }}$ Street, New York, NY 10021, USA

$\dagger$ Electronic supplementary information (ESI) available. See DOI: 10.1039/d0ra07482a

\$ They equally contributed to this work.
}

structure in high aspect ratios. ${ }^{11-13}$ The chemical etching method typically requires specialized equipment to react the precursors in high temperature, controlled atmosphere and pressure conditions, increasing production costs. ${ }^{14}$

One of the most effective and versatile sacrificial template methods for synthesizing hollow metal nanoparticles with controllable composition and size is galvanic replacement. ${ }^{6,15}$ Galvanic replacement is a corrosion process that is driven by the difference in reduction potentials between a metallic substrate and metal ions in solution. ${ }^{1}$ The advantage of utilizing the galvanic mechanism for nanomaterial synthesis is that the resulting hollow nanoparticles can be rationally designed by using precursors that have staggered reduction potentials in their exchange reactions. Upon contact between such metal substrate and ions in solution, the one with lower reduction potential is oxidized, while the other with higher reduction potential is reduced, and as a result, metal ions from solution are plated onto the template. ${ }^{1,14}$

As these metals are exchanged, the final product typically possesses a shape similar to the original substrate, but the element of substrate is then swapped to metal ions from solution. Recently, element exchange triggered by surface energy difference has been applied to engineer various hollow metal nanoparticles, ${ }^{16}$ and Xia group has pioneered the application of galvanic exchange reactions in the synthesis of $\mathrm{Au}$, Pt and $\mathrm{Pd}$ hollow metal nanoparticles from Ag nanoparticle substrates. ${ }^{17,18}$ However, this approach has rarely been applied for the synthesis of 1D metal oxide hollow nanostructures starting from sacrificial silver nanowire substrates.

Here, we demonstrate for the first time the synthesis of hollow iron oxide nanotubes starting from silver nanowires using one-pot, two-step galvanic replacement reactions. It should be noted that direct replacement of $\mathrm{Ag}$ to $\mathrm{Fe}_{2} \mathrm{O}_{3}$ is not 
viable considering both species need to oxidize simultaneously, and that is our motivation for developing the one-pot, double galvanic exchange reactions of nanotubes. This method can also be generalized for rational synthesis of other metal oxide nanotubes as the formation is predicted by redox potentials of involved ion species.

Fig. 1 summarizes the synthesis scheme of iron oxide nanotubes starting from silver nanowires as substrate. Here, it should be noted that the reactions are broken down into two steps for the ease of understanding the reaction mechanism, while all of these reactions are done in one pot. The galvanic replacement reaction is driven by the differences in reduction potentials between the two species involved. ${ }^{15}$ In Fig. $1 \mathrm{a}, \mathrm{Ag}$ is dissolved into solution and electrons through $\mathrm{Ag}$ oxidation reduce $\mathrm{MnO}_{4}{ }^{-}$ions in solution to $\mathrm{Mn}_{3} \mathrm{O}_{4}$. The replacement to $\mathrm{Mn}_{3} \mathrm{O}_{4}$ in nanotubes takes place due to the redox potential landscape between $\mathrm{Ag}$ and $\mathrm{MnO}_{4}{ }^{-}$(Fig. 1c) since the absolute value of reduction potential of $\mathrm{MnO}_{4}{ }^{-}(1.47 \mathrm{~V})$ is larger than the one for $\mathrm{Ag}(-0.8 \mathrm{~V})$ as shown in Fig. 1c. ${ }^{19}$ This first galvanic replacement reaction was initiated by mixing silver nanowires $\left(5 \mathrm{mg} \mathrm{mL}{ }^{-1}, 3 \mathrm{~mL}\right)$ of $20 \mathrm{~nm}$ in diameter dispersed in water with an aqueous solution of potassium permanganate $(1 \mathrm{mM}, 18.95$ $\mathrm{mL}$ ) and subsequently heating the reaction mixture to $100{ }^{\circ} \mathrm{C}$ for 40 minutes. ${ }^{19}$ At this point, the shape of the original nanowire (Fig. 2a) has already transformed into a hollow nanotube (Fig. 2b).

In the second galvanic exchange reaction (Fig. 1b), $\mathrm{Mn}_{3} \mathrm{O}_{4}$ nanotubes from galvanic reaction 1 are transformed to $\mathrm{Fe}_{2} \mathrm{O}_{3}$ nanotubes as $\mathrm{Mn}$ ions are replaced by $\mathrm{Fe}$ ions. The redox

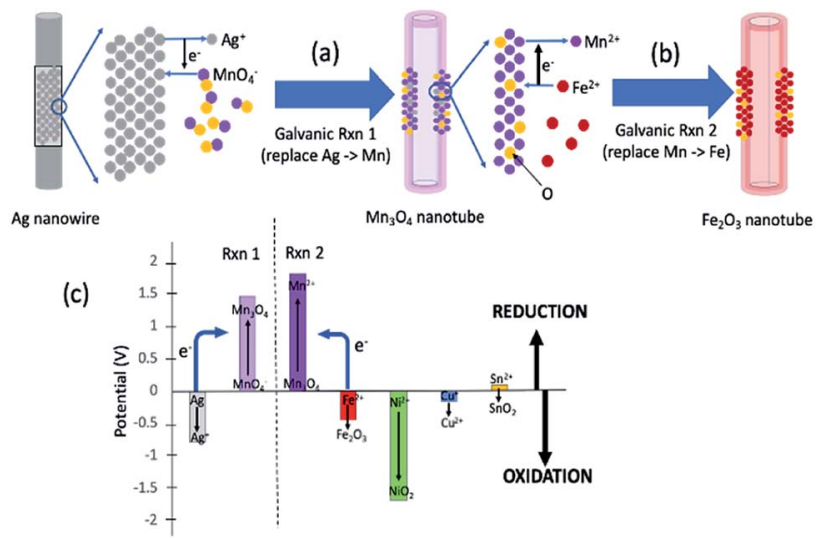

Fig. 1 General concept of one-pot double galvanic exchange reactions for the synthesis of iron oxide nanotubes. (a) The first galvanic exchange reaction to generate $\mathrm{Mn}_{3} \mathrm{O}_{4}$ nanotubes from Ag nanowire substrate. (b) The second galvanic exchange reaction to replace $\mathrm{Mn}$ to Fe nanotubes. (c) Reduction potential landscape for iron oxide and other possible metal oxide in nanotube formation (half-reactions and their electric reduction potentials are also shown in ESI Table S-1†). When the reduction potential of one element is larger than the other, the element with lower reduction potential (e.g., Fe) can donate its electrons to reduce $\mathrm{Mn}_{3} \mathrm{O}_{4}$, which has a larger reduction potential (see blue arrows for the direction of electron flow). A variety of metal oxide nanotubes in addition to iron oxide can be formed when the final element of the nanotube is oxidized due to the large reduction potential of the intermediate $\mathrm{Mn}_{3} \mathrm{O}_{4}$ nanotube.
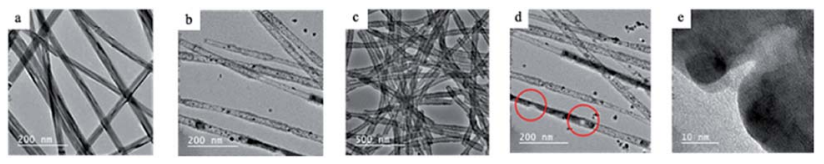

Fig. 2 TEM images of (a) Ag nanowire substrates, (b) $\mathrm{Mn}_{3} \mathrm{O}_{4}$ nanotubes, (c) $\mathrm{Fe}_{2} \mathrm{O}_{3}$ nanotubes, (d) a section of $\mathrm{Mn}_{3} \mathrm{O}_{4}$ nanotubes where pinholes were observed on the surface (red circles), (e) pinholes in (d) with a higher magnification.

potential difference is aligned favourably for the oxidation of $\mathrm{Fe}$ ions $(-0.77 \mathrm{~V})$ via the reduction of $\mathrm{Mn}$ ions $(1.82 \mathrm{~V})$ (Fig. 1c) during this process. $\mathrm{Fe}_{2} \mathrm{O}_{3}$ nanotubes were generated when $\mathrm{Mn}_{3} \mathrm{O}_{4}$ nanotubes were mixed with iron(II) perchlorate solution $\left(1 \mathrm{mg} \mathrm{mL}{ }^{-1}, 12 \mathrm{~mL}\right.$ ) at $80^{\circ} \mathrm{C}$ for 2 hours. The resulting sample is then centrifuged at $3000 \mathrm{~g}$ for 10 minutes, and the pellet is resuspended in deionized water for analysis. Transmission electron microscopic (TEM) image of $\mathrm{Fe}_{2} \mathrm{O}_{3}$ nanotubes (Fig. 2c) reveals that the diameter of nanotubes increases from $27.0 \pm$ $2.0 \mathrm{~nm}$ in $\mathrm{Mn}_{3} \mathrm{O}_{4}$ nanotubes to $40.0 \pm 3.0 \mathrm{~nm}$ in $\mathrm{Fe}_{2} \mathrm{O}_{3}$ nanotubes. The elemental mapping of energy-dispersive X-ray spectroscopy (EDXS) was also applied to analyze the elemental replacement in the process of one-pot galvanic reaction in Fig. 1. When intermediate products of nanotubes after galvanic reaction 1 (Fig. 3a) were probed by EDXS mapping with scanning transmission electron microscopy (STEM), the composition was mostly Mn (Fig. 3c) and O (Fig. 3d) with minimal leftover Ag substrate (Fig. $3 \mathrm{~b}$ ). The second galvanic exchange reaction generated $\mathrm{Fe}_{2} \mathrm{O}_{3}$ nanotubes (Fig. 3e) in high yield and $\mathrm{Mn}$ ions were successfully exchanged by Fe ions (Fig. 3i), and the resulting nanotubes were almost free from $\mathrm{Ag}$ (Fig. 3f) and Mn (Fig. 3g).

When the surface structure of $\mathrm{Mn}_{3} \mathrm{O}_{4}$ nanotubes was examined by high resolution TEM (HRTEM), pinholes on the sidewalls of $\mathrm{Mn}_{3} \mathrm{O}_{4}$ nanotubes were revealed where the dark contrast of remaining $\mathrm{Ag}$ inside make these holes more visible (inside red circles in Fig. 2d). These pinholes are even more evident in a HRTEM image in Fig. 2e. This observation led to the hypothesis that the hollowing process of template nanowires could occur through the pinhole dissolution mechanism. Pinhole corrosion is the process where pinholes serve as paths
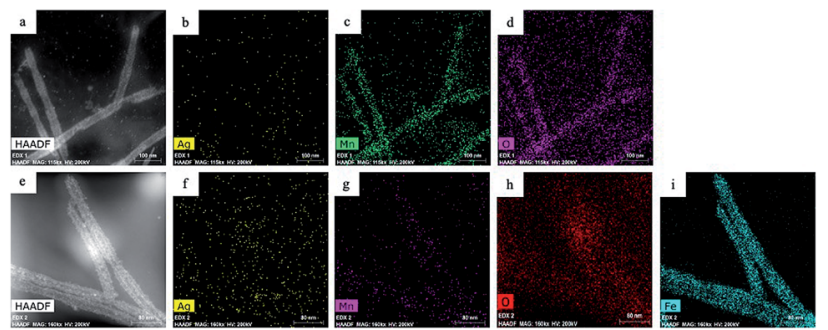

Fig. 3 (a) Scanning transmission electron microscopic (STEM) image of $\mathrm{Mn}_{3} \mathrm{O}_{4}$ nanotubes and corresponding elemental mapping in (b) Ag, (c) $\mathrm{Mn}$, and (d) $\mathrm{O}$ with EDXS. (e) STEM image of $\mathrm{Fe}_{2} \mathrm{O}_{3}$ nanotubes and corresponding elemental mapping in (f) $\mathrm{Ag}$, (g) $\mathrm{Mn}$, (h) $\mathrm{O}$, and (i) Fe with EDXS 
of material transport during the dissolution of the core of template nanoparticles in the course of the redox reactions. ${ }^{15}$ In this mechanism, the stoichiometry of redox reaction is important to determine the degree of atomic replacement and hollowing process which in turn affects the nanotube's composition and sidewall thickness. ${ }^{20}$ In the hollowing process of $\mathrm{Ag}$ nanowires, the $\mathrm{Ag} \rightarrow \mathrm{Mn}$ replacement reaction is;

$$
16 \mathrm{H}_{(\mathrm{aq})}^{+}+3 \mathrm{MnO}_{4(\mathrm{aq})}{ }^{-}+\underset{\underset{8}{8 \mathrm{H}_{2} \mathrm{O}_{(\mathrm{l})}}}{+13 \mathrm{Ag}_{(\mathrm{s})}} \rightarrow \mathrm{Mn}_{3} \mathrm{O}_{4(\mathrm{~s})}+13 \mathrm{Ag}_{(\mathrm{aq})}^{+}+
$$

In this reaction, one $\mathrm{Mn}_{3} \mathrm{O}_{4}$ is produced at the expense of $13 \mathrm{Ag}$ atoms based on the stoichiometric ratio above. Such high turnover of $\mathrm{Ag}$ replacement through the efficient pinhole dissolution paths should guide the redox reaction to nearly complete replacement, which is difficult to be accomplished solely by room temperature Kirkendall effect (i.e., the diffusion of $\mathrm{Ag}$ from template nanowires is slow at relatively low reaction temperature). ${ }^{21}$ In addition, the atomic\% ratio of $\mathrm{Ag}$ versus $\mathrm{Mn}$ of the $\mathrm{Mn}_{3} \mathrm{O}_{4}$ nanotubes is $0.1: 1.0$ by the STEM-EDX mappings of Fig. $3 \mathrm{~b}$ and $\mathrm{c}$, showing efficient $\mathrm{Ag}$ atom replacement. This result also supports that the transformation from $\mathrm{Ag}$ nanowire to $\mathrm{Mn}_{3} \mathrm{O}_{4}$ nanotubes is mainly driven by the pinhole dissolution mechanism and that the Kirkendall effect may play only a minor role in the replacement reaction. ${ }^{15}$ Based on TEM images, the wall thickness of $\mathrm{Mn}_{3} \mathrm{O}_{4}$ nanotubes is $5.1 \pm 2.0 \mathrm{~nm}(n=23)$ (Fig. 2b) after $\mathrm{Ag}$ nanowire templates with diameters of $20 \pm$ $2.0 \mathrm{~nm}$ (Fig. 2a) are consumed, which is consistent with the previous observations about the degree of sidewall thickness with respect to the diameter of templates with similar stoichiometry in their replacement reactions. ${ }^{\mathbf{2 0 , 2 2}}$ Further atomic replacement from $\mathrm{Mn}$ to Fe occurs by the reaction;

$8 \mathrm{H}_{(\mathrm{aq})}{ }^{+}+\mathrm{Mn}_{3} \mathrm{O}_{4(\mathrm{~s})}+2 \mathrm{Fe}_{(\mathrm{aq})}{ }^{2+} \rightarrow 3 \mathrm{Mn}_{(\mathrm{aq})}{ }^{2+}+4 \mathrm{H}_{2} \mathrm{O}_{(\mathrm{l})}+2 \mathrm{Fe}_{(\mathrm{s})}{ }^{3+}$

where two Fe ions are produced by replacing one $\mathrm{Mn}_{3} \mathrm{O}_{4}$. Since numerous lattice vacancies would be formed on the sidewall of $\mathrm{Mn}_{3} \mathrm{O}_{4}$ nanotubes (due to high atomic replacement ratio of $\mathrm{Ag}$ to-Mn in $13: 1$ ) and the possibility of these vacancies to coalesce to form pinholes, ${ }^{23}$ the replacement from $\mathrm{Mn}$ to $\mathrm{Fe}$ is efficient through pinholes. Such processes will further deplete the amount of $\mathrm{Ag}$ in the resulting $\mathrm{Fe}_{2} \mathrm{O}_{3}$ nanotubes, thus, it is reasonable to observe almost complete replacement of $\mathrm{Ag}$ in $\mathrm{Fe}_{2} \mathrm{O}_{3}$ nanotubes with atomic\% ratio of $\mathrm{Ag}$ versus $\mathrm{Fe}$ of $0.006: 1$ from Fig. $3 \mathrm{f}$ and i. Moreover, due to the atomic ratio of this replacement, it is plausible to observe the wall thickness of $\mathrm{Fe}_{2} \mathrm{O}_{3}$ nanotubes to increase to $11.2 \pm 3.2 \mathrm{~nm}(n=23)$ (Fig. 2c).

Another supporting observation for the pinhole dissolution mechanism is that the volume of voids inside $\mathrm{Ag}$ nanowires increases during the transformation to $\mathrm{Mn}_{3} \mathrm{O}_{4}$ nanotubes with the concentration of $\mathrm{Mn}$ precursor. When various concentrations of $\mathrm{KMnO}_{4}$ were reacted with $\mathrm{Ag}$ nanowire solution, a series of TEM images in Fig. S1† showed that void formation is expanded by increasing the amount of $\mathrm{KMnO}_{4}$. Since more $\mathrm{Mn}$ ions consume $\mathrm{Ag}$ ions with efficient atomic replacement through pinholes, these TEM images support the pinhole dissolution mechanism as a major pathway. In summary, all evidence here supports that pinhole dissolution is the main mechanism for the synthesis of $\mathrm{Fe}_{2} \mathrm{O}_{3}$ nanotubes from $\mathrm{Ag}$ nanowire templates.

Since manganese possesses a very high reduction potential of $1.82 \mathrm{~V}$, it is feasible to be utilized as a template for a variety of metal oxide nanotubes. Thus, this enables the design of a variety of metal oxide nanotube syntheses, as long as the replacing metal ions can be oxidized and have reduction potentials smaller than $1.82 \mathrm{~V}$ (in absolute values). Due to the high reduction potential of $\mathrm{Mn}$ ions, there are many metal ions that align with this reduction potential hierarchy for the second galvanic replacement as shown in Fig. 1c. To test the generality of this one-pot, double galvanic method for metal oxide nanotube synthesis, we examined the synthesis of $\mathrm{NiO}_{2}, \mathrm{CuO}$, and $\mathrm{SnO}_{2}$ nanotubes from $\mathrm{Ag}$ nanowire substrates. In terms of the redox potential landscape, all of the reduction potentials are appropriate for one-pot double galvanic exchange as the reduction potential of $\mathrm{NiO}_{2} / \mathrm{Ni}^{2+}, \mathrm{SnO}_{2} / \mathrm{Sn}^{2+}$ and $\mathrm{Cu}^{2+} / \mathrm{Cu}^{+}$is $1.68 \mathrm{~V},-0.094 \mathrm{~V}$, and $0.161 \mathrm{~V}$, respectively. ${ }^{24,25}$ In theory, these ions can reduce $\mathrm{Mn}_{3} \mathrm{O}_{4}(1.82 \mathrm{~V})$ during the second galvanic replacement reaction because their reduction potentials in absolute values are lower than $\mathrm{Mn}_{3} \mathrm{O}_{4}$. In this trial, tin(II) chloride, copper(I) chloride, and nickel(II) chloride were used as precursor solutions to synthesize $\mathrm{SnO}_{2}, \mathrm{CuO}$, and $\mathrm{NiO}_{2}$ nanotubes. TEM images (Fig. 4a, d and g) indicate that all of these nanotubes possess a hollow structure with an average diameter of $40 \mathrm{~nm}$, suggesting that their size was controlled by the size of $\mathrm{Ag}$ nanowire substrate. The elemental transformation of template nanowires to target nanotubes was confirmed by EDXS maps in Fig. 4b, c, e, f, h and i.
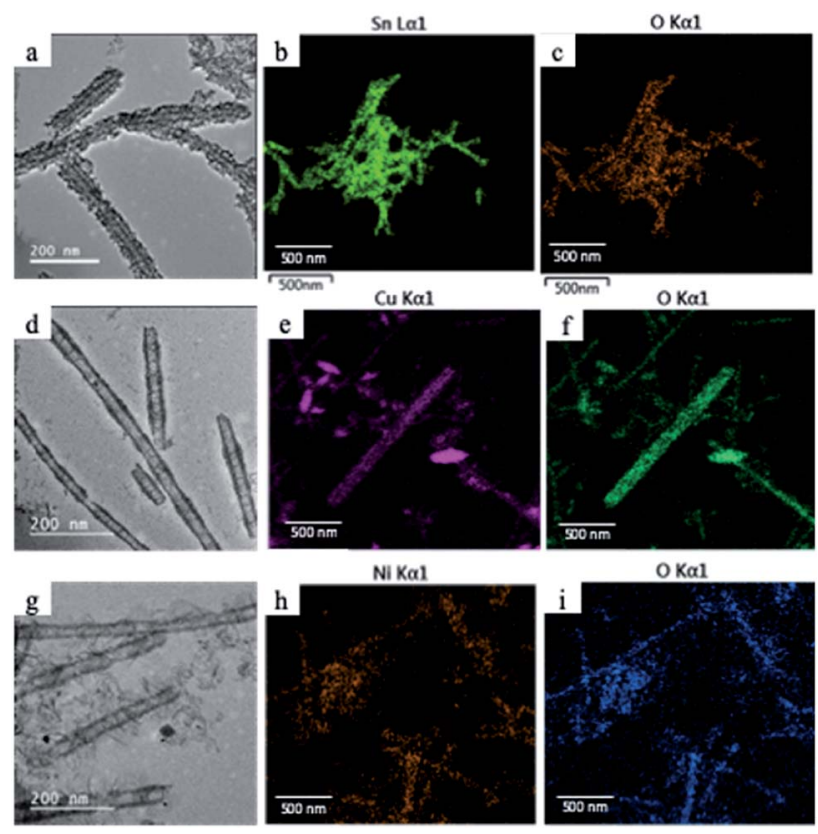

Fig. 4 (a) TEM image of $\mathrm{SnO}_{2}$ nanotubes and elemental mapping of (b) $\mathrm{Sn}$ and (c) $\mathrm{O}$ with EDXS. (d) TEM image of $\mathrm{CuO}$ nanotubes and elemental mapping of (e) $\mathrm{Cu}$ and ( $f$ ) $\mathrm{O}$ with EDXS. (g) TEM image of $\mathrm{NiO}_{2}$ nanotubes and elemental mapping of (h) $\mathrm{Ni}$ and (i) $\mathrm{O}$ with EDXS. 
In conclusion, we combined two galvanic reactions in onepot for the synthesis of iron oxide nanotubes from commercially available $\mathrm{Ag}$ nanowires. The large reduction potential of the $\mathrm{Mn}_{3} \mathrm{O}_{4}$ nanotube intermediate opens this method to final exchange by various metal ions to form many different metal oxide nanotubes. We have demonstrated it by the synthesis of other metal oxide nanotubes such as $\mathrm{SnO}_{2}, \mathrm{CuO}$, and $\mathrm{NiO}_{2}$, which have favorable staggered redox potential arrangement with $\mathrm{Mn}_{3} \mathrm{O}_{4}$ reduction. This generalized double galvanic replacement approach will offer robust, economical, and scaleup engineering of a variety of hollow one-dimensional metal oxide nanostructures.

\section{Conflicts of interest}

There are no conflicts to declare.

\section{Note added after first publication}

This article replaces the version published on 20 October 2020, which contained errors in Fig. 3.

\section{Acknowledgements}

This work was supported by the National Institute on Minority Health and Health Disparities (NIMHD) of NIH (MD007599) for nanoparticle analysis. Various nanotube fabrications were supported by NIH/NCATS (UL1TR002384). AP thank Dr Jorge Morales (CCNY-CUNY) and Dr Sheng Zhang (ASRC-CUNY) for assistance in TEM/EDXS analysis of these nanotubes.

\section{References}

1 X. Xiaohu, Y. Wang, A. Ruditskiy and Y. Xia, Adv. Mater., 2013, 25, 6313-6333.

2 T. Zhai, X. S. Fang, M. Y. Liao, X. Xu, H. Zeng, B. Yoshio and

D. Golberg, Sensors, 2009, 9, 6504-6529.

3 S. Dang, Q. L. Zhu and Q. Xu, Nat. Rev. Mater., 2017, 2017, 17075.

4 X. Wang, J. Feng, Y. Bai, Q. Zhang and Y. Yin, Chem. Rev., 2016, 116, 10983-11060.
5 K. An and T. Hyeon, Nano Today, 2009, 4, 359-373.

6 A. G. M. Da Silva, T. S. Rodrigues, S. J. Haigh and P. H. C. Camargo, Chem. Commun., 2017, 53, 7135-7148.

7 W. Wei, Z. Wang, L. Zhuang, L. Yang, H. Liang, C. Dezhi, A. Umarde, G. Lin and L. Jinghong, J. Power Sources, 2013, 238, 376-387.

8 J. H. Lee, Sens. Actuators, B, 2009, 140, 319-336.

9 H. C. Zeng, J. Mater. Chem., 2006, 16, 649-662.

10 S. F. Soares, T. Fernandes, A. L. Daniel-Da-Silva and T. Trindade, Proc. R. Soc. A, 2019, 475, 20180677.

11 C.-J. Jia, L.-D. Sun, Z.-G. Yan, L.-P. You, F. Luo, X.-D. Han, Y.-C. Pang, Z. Zhang and C.-H. Yan, Adv. Mater., 2005, 117, 4402-4407.

12 W. Wu, X. Xiao, S. Zhang, J. Zhou, L. Fan, F. Ren and C. Jiang, J. Phys. Chem. C, 2010, 114, 16092-16103.

13 Z.-G. Yue, W. Wei, Z.-X. You, Q.-Z. Yang, H. Yue, Z.-G. Su and G.-H. Ma, Adv. Funct. Mater., 2011, 21, 3446-3453.

14 S. W. Chee, S. F. Tan, Z. Baraissov, M. Bosman and U. Mirsaidov, Nat. Commun., 2017, 8, 1224.

15 M. H. Oh, T. Yu, S.-H. Yu, B. Lim, K.-T. Ko, M.-G. Willinger, D.-H. Seo, B. H. Kim, M. G. Cho, j.-H. Park, K. Kang, Y.-E. Sung, N. Pinna and T. Hyeon, Science, 2013, 340, 964968.

16 Z. Wei and H. Matsui, Nat. Commun., 2014, 5, 3870.

17 Y. G. Sun, B. T. Mayers and Y. N. Xia, Nano Lett., 2002, 2, 481485.

18 Y. G. Sun, B. T. Mayers and Y. N. Xia, Adv. Mater., 2003, 15, 641-646.

19 H. Dong, E. K. Koh and S. Y. Lee, J. Nanosci. Nanotechnol., 2009, 9, 6511-6517.

20 Y. G. Sun, B. T. Mayers and Y. N. Xia, Nano Lett., 2002, 2, 481485.

21 J. Erlebacher and D. Margetis, Phys. Rev. Lett., 2014, 112, 155505.

22 Y. G. Sun and Y. N. Xia, Science, 2002, 298, 2176-2179.

23 Y. G. Sun and Y. N. Xia, Nano Lett., 2003, 3, 1569-1572.

24 S. G. Bratsch, J. Phys. Chem. Ref. Data, 1989, 18, 1-21.

25 A. Bard, R. Parsons and J. Jordan, Standard potentials in aqueous solution, Marcel Dekker, Inc., New York, 1985. 